\title{
Impact of the Stratosphere on the Winter Tropospheric Teleconnections between ENSO and the North Atlantic and European Region
}

\author{
CHIARA CAGNAZZO \\ Centro Euro-Mediterraneo per i Cambiamenti Climatici, Bologna, Italy \\ ELISA MANZINI
}

Istituto Nazionale di Geofisica e Vulcanologia, and Centro Euro-Mediterraneo per i Cambiamenti Climatici, Bologna, Italy

(Manuscript received 27 March 2008, in final form 21 August 2008)

\begin{abstract}
The possible role of stratospheric variability on the tropospheric teleconnection between El NiñoSouthern Oscillation (ENSO) and the North Atlantic and European (NAE) region is addressed by comparing results from two ensembles of simulations performed with an atmosphere general circulation model fully resolving the stratosphere (with the top at $0.01 \mathrm{hPa}$ ) and its low-top version (with the top at $10 \mathrm{hPa}$ ). Both ensembles of simulations consist of nine members, covering the 1980-99 period and are forced with prescribed observed sea surface temperatures. It is found that both models capture the sensitivity of the averaged polar winter lower stratosphere to ENSO in the Northern Hemisphere, although with a reduced amplitude for the low-top model. In late winter and spring, the ENSO response at the surface is instead different in the two models. A large-scale coherent pattern in sea level pressure, with high pressures over the Arctic and low pressures over western and central Europe and the North Pacific, is found in the FebruaryMarch mean of the high-top model. In the low-top model, the Arctic high pressure and the western and central Europe low pressure are very much reduced. The high-top minus low-top model difference in the ENSO temperature and precipitation anomalies is that North Europe is colder and the Northern Atlantic storm track is shifted southward in the high-top model. In addition, it has been found that major sudden stratospheric warming events are virtually lacking in the low-top model, while their frequency of occurrence is broadly realistic in the high-top model. Given that this is a major difference in the dynamical behavior of the stratosphere of the two models and that these events are favored by ENSO, it is concluded that the occurrence of sudden stratospheric warming events affects the reported differences in the tropospheric ENSO-NAE teleconnection. Given that the essence of the high-top minus low-top model difference is a more annular (or zonal) pattern of the anomaly in sea level pressure, relatively larger over the Arctic and the NAE regions, this interpretation is consistent with the observational evidence that sudden stratospheric warmings play a role in giving rise to persistent Arctic Oscillation anomalies at the surface.
\end{abstract}

\section{Introduction}

There is growing evidence that the state and the variability of the lower stratosphere affect tropospheric climate. Observational works have shown that large perturbations of the Northern Hemisphere polar vortex are associated with a seesaw in surface pressure encompassing the entire high latitudes (the Arctic Oscillation, AO; Baldwin and Dunkerton 2001; Thompson and

Corresponding author address: Chiara Cagnazzo, Centro EuroMediterraneo per i Cambiamenti Climatici, Viale Aldo Moro 44, 40127 Bologna, Italy.

E-mail: cagnazzo@bo.ingv.it
Wallace 2000). Moreover, since the pioneering work of Boville (1984), it is known that large biases in the modeling of the lower-stratospheric polar-night jet have an impact on aspects of the modeled tropospheric circulation, such as stationary planetary waves and even smaller-scale transient eddies. More recently, additional evidence has been reported. For instance, Norton (2003) showed that the persistence of the surface Arctic Oscillation is sensitive to the variability of the stratospheric polar vortex. Charlton et al. (2004) used a weather forecast model and have found that the transient response of the troposphere, on time scales between 10 and 20 days, is sensitive to the stratospheric initial conditions. Scaife et al. (2005) applied an experimental design similar to 
the one of Norton (2003) and found that an imposed strengthening of the polar vortex during the boreal winter over about $30 \mathrm{yr}$ can induce a positive trend in the North Atlantic Oscillation. Although these modeling works (as well as others) varied greatly in design and time scales considered, they all tend to show that the averaged influence of the stratosphere on the troposphere [as depicted by the sea level pressure (SLP) or the 1000-hPa geopotential height] has a large-scale quasiannular or quasi-zonal pattern, generally more pronounced over the North Atlantic sector. The construction of a comprehensive theory for the downward propagation of the stratospheric disturbances is a topic of current research, and several mechanisms have been proposed (Song and Robinson 2004 and reference therein; Thompson et al. 2006).

Despite growing evidence of the effect of stratospheric dynamics on surface conditions in the development of climate (coupled atmosphere-ocean) models, the vertical resolution and location of the model top have hardly received any attention (Kushner et al. 2007).

The purpose of this work is to study the possible role of stratospheric variability in the tropospheric teleconnection between El Niño-Southern Oscillation (ENSO) and the North Atlantic and European (NAE) region. The mechanisms responsible for the remote influence of ENSO on the North Atlantic and European region are still controversial and certainly indirect. The "canonical" teleconnection that emerged from observations (Fraedrich and Müller 1992, among others) consists of a cold and dry anomaly over northern Europe, high sea level pressure over Scandinavia and low sea level pressures over central and western Europe, and increased precipitation over Southern Europe. Brönnimann (2007) reported that the ENSO teleconnection over Europe may also depend on the seasons and that it is more substantial for strong ENSO events, for instance the one that occurred in 1940-42. The extensive literature on the mechanism behind the ENSO-NAE teleconnection has so far mostly focused on the role of tropospheric downstream effects emanating from the North Pacific (Hoerling et al. 1997; Honda et al. 2001, among others) and on the role of tropical Atlantic and Indo-Pacific sea surface temperatures (SSTs; Mathieu et al. 2004, among others). These mechanisms are discussed in the recent review of the ENSO effects on Europe by Brönnimann (2007), where also the possible role of the stratosphere is reported.

The motivation to investigate a stratospheric role in the ENSO teleconnections is given by 1) the evidence of the influence of ENSO on the stratospheric polar vortex (Manzini et al. 2006, and references therein) and 2) the propensity for the possible downward influence of the stratosphere to be realized in the NAE region. Our objective is, therefore, also relevant for the broader topic of determining the type of atmospheric models (specifically, the vertical resolution and location of model top) most appropriate for seasonal forecast at high and middle latitudes, because of the dependence of the predictability of the North Atlantic and European winter climate on ENSO (Mathieu et al. 2004). However, comparing the role of the stratosphere to other processes that may be involved in the ENSO-NAE teleconnection is a rather comprehensive topic, beyond the scope of the present investigation.

In this work, we compare results from two ensembles of simulations performed with atmosphere general circulation models with prescribed observed sea surface temperatures. The first ensemble is obtained from a stratosphere-resolving atmosphere general circulation model (results reported in Manzini et al. 2006). The second ensemble is obtained from a state-of-the art atmospheric model-such as those used in climate models (coupled atmosphere-ocean models; Randall et al. 2007) — with a top in the middle stratosphere. The latter model can, therefore, be considered as a benchmark model of the current state of modeling the stratosphere in climate research. Because the two models considered are closely connected, as described below, the comparison of the two ensembles of simulations gives insights in to the impact of the modeling of the full stratosphere.

The model results of Manzini et al. (2006) showed that the northern lower-stratospheric response to ENSO is characterized by a polar warming of a few degrees in winter and early spring. These results are broadly consistent with the later reported estimate from observations of Camp and Tung (2007) as well as earlier works. In Manzini et al. (2006), the modeled stratospheric response is robust and large scale enough in the ensemble mean to emerge also in the zonal mean and to propagate to the surface. Therefore, Manzini et al. (2006) suggested that there could be a downward feedback of the ENSO stratospheric response to the surface in late winter and early spring at northern middle and high latitudes. Evidence for this downward feedback is presented here by comparing modeled tropospheric meteorological fields in the two ensembles of simulations done with and without a well-resolved stratosphere, respectively. In addition, this work extends the Manzini et al. (2006) results of the stratospheric response to ENSO by analyzing the occurrence of sudden stratospheric warming events and their dependency on ENSO in both models.

In summary, a case of a stratospheric polar vortex modified by upward propagating tropospheric disturbances (troposphere-stratosphere connection favored 
by ENSO conditions) is used to investigate if the following large-scale disturbances of the polar vortex, manifested as zonal mean anomalies, can feed back on to the tropospheric climate. Therefore, the current investigation is also an attempt to extract the tropospheric response of the troposphere-stratosphere-troposphere events discussed by Reichler et al. (2005), in a less idealized and less controlled set of numerical experiments.

This paper is structured as follows: methodology and models are described in section 2. Results from the two ensembles of simulations are reported in section 3 , and a discussion and conclusions are presented in section 4 .

\section{Methodology}

The ensemble of simulations reported in Manzini et al. (2006) was performed with the MAECHAM5 general circulation model (hereafter "high top" model) with vertical domain ranging from the surface to 0.01 $\mathrm{hPa}$ and 39 vertical levels.

Here the results from the Manzini et al. (2006) ensemble are compared to an analogous ensemble of 9 simulations performed instead with the standard ECHAM5 model (hereafter "low top" model) with vertical domain ranging from the surface to $10 \mathrm{hPa}$ and 19 vertical levels.

For details on the ECHAM5 general circulation model, refer to Roeckner et al. (2006). The horizontal resolution employed in both the high- and low-top models is T42. The two models share the same representation of tropospheric physics, including orographic gravity wave drag parameterization and its parameter setting, and the same vertical resolution from the surface to $500 \mathrm{hPa}$. This design is targeted to minimize the difference in the results from the models as a result of the representation of the lower troposphere. Given that the top of the ECHAM5 model is at $10 \mathrm{hPa}$, the ECHAM5 model includes only a partial representation of the stratosphere with respect to the MAECHAM5 version for the following reasons: (i) ECHAM5 has lower vertical resolution in the upper troposphere and lower stratosphere (see Table 1); (ii) ECHAM5 has relatively large dissipation (sponge layer), applied between 50 and $10 \mathrm{hPa}$, and realized by lowering the order of the horizontal diffusion operator and by increasing its strength. The well-known justification of the sponge layer is to reduce reflection from upward propagating waves and to obtain a realistic mean state. However, stratospheric variability is also removed. The MAECHAM5 model is, therefore, less dissipative between 50 and $10 \mathrm{hPa}$ than the ECHAM5 model, and planetary waves are free to propagate upward above $50-10 \mathrm{hPa}$ and interact with the mean stratospheric flow in the
TABLE 1. Number of levels and their average resolution $(\Delta Z)$ for the low-top (ECHAM5) and high-top (MAECHAM5) models.

\begin{tabular}{lcccc}
\hline \hline & $\begin{array}{c}500-100 \mathrm{hPa} \\
\text { No. of } \\
\text { levels }\end{array}$ & $\begin{array}{c}500-100-\mathrm{hPa} \\
\Delta \boldsymbol{Z}(\mathrm{km})\end{array}$ & $\begin{array}{c}\text { No. of } \\
\text { levels }\end{array}$ & $\begin{array}{c}100-10-\mathrm{hPa} \\
\Delta Z(\mathrm{~km})\end{array}$ \\
\hline ECHAM5 & 6 & 2 & 4 & 4.6 \\
MAECHAM5 & 7 & 1.4 & 9 & 1.9
\end{tabular}

MAECHAM5 model only. A further difference is the inclusion of the nonorographic gravity wave drag only in the MAECHAM5 model (see Manzini et al. 2006 and references therein). Given that the implementation of the nonorographic gravity wave drag is momentum conserving and that the orographic gravity wave drag is depleted within the model domain in MAECHAM5, spurious downward influences (Shaw and Shepherd 2007) can be excluded by design in MAECHAM5. Instead, the low-top ECHAM5 model can be affected by such influences. This aspect of the ECHAM5 model can be considered as another possible deficiency in design caused by the limited representation of the stratosphere in current climate models.

In the following section, we compare results from two ensembles of simulations: one ensemble performed with a high-top model and the other with a low-top model. For each ensemble there are nine simulations, and each simulation is performed with the prescribed observed monthly-mean SST and sea ice concentration [Atmospheric Model Intercomparison Project II dataset (AMIPII) from the Program for Climate Model Diagnosis and Intercomparison (available online at http:// www-pcmdi.llnl.gov/projects/amip)] for the 20-yr period 1980-99 (after 2 yr of spinup for each simulation). Within each ensemble, each simulation differs only for the initial conditions, taken from a 20-yr control simulation with climatological SST and sea ice cover (SIC) performed with the respective model. In both models, greenhouse gases are held fixed at present values. A monthly zonal mean climatological ozone distribution (Fortuin and Kelder 1998) is used.

The statistical comparison between the two ensembles is limited to the warm ENSO events, extracted following the composite approach described in Manzini et al. (2006). For each month of the extended winter season (October-April), warm ENSO, neutral, and cold ENSO composites of monthly means are constructed from the ensemble monthly-mean time series. Both the warm and cold ENSO composites are made of the four largest events that occurred in the period 1980-99. The neutral composite is made of the $11 \mathrm{yr}$ within the 198099 period that exclude both warm and cold ENSO 
events (refer to Manzini et al. (2006) for further details). Given the nonlinearity of the tropospheric teleconnections related to ENSO events (Hoerling et al. 1997), the warm ENSO anomalies are defined as the difference: warm ENSO composite minus the neutral composite. Given that in the following study only the warm ENSO anomaly is considered, the "warm" specification is omitted.

The analysis is restricted to the warm ENSO events because Manzini et al. (2006) showed that the stratosphere response is negligible for the cold ENSO events, at least for those occurred during the 1980-99 decades.

In the polar lower stratosphere, the mean climate of MAECHAM5 tends to be warmer than that of ECHAM5 during northern winter (at the North Pole at $10 \mathrm{hPa}$, up to $10-14 \mathrm{~K}$ ). These differences are localized in the sponge region of the ECHAM5 model and are expected to be due to the dissipation differences in the two models. In the troposphere, these differences are instead less than $1 \mathrm{~K}$. Close to the surface, the mean surface climate of the two models is constrained to be the same by the prescription of the identical sea surface temperatures and sea ice concentrations.

\section{Results}

\section{a. ENSO signal in the winter stratosphere}

The influence on the zonal mean winter stratospheric circulation of ENSO events found in Manzini et al. (2006) is reported here in Fig. 1 (left panels). In addition, Fig. 1 shows the ENSO anomalies for the zonal mean temperature at $80^{\circ} \mathrm{N}$ and zonal mean zonal wind at $60^{\circ} \mathrm{N}$ for the low-top (ECHAM5) model (right panels). The comparison of the high-top model with 40-yr European Centre for Medium-Range Weather Forecasts Re-Analysis (ERA-40) is shown in Manzini et al. (2006) and is not reported here. Their results indicated that the high-top model captures the behavior of the ENSO anomaly emerging from the ERA-40 data.

In February, the peak anomaly in zonal mean temperature at $10 \mathrm{hPa}$ is comparable in the two models (about $4 \mathrm{~K}$ ). However, at $100 \mathrm{hPa}$ the February temperature anomaly in the low-top model is half the size (Fig. 2, upper left). Below the sponge region of the lowtop model, the high minus top model difference in zonal mean temperature is largest (about $2 \mathrm{~K}$ ) between 100 and $200 \mathrm{hPa}$ in February and March (FM). The weakening of the polar vortex that occurs in February and March in the lower stratosphere is clearly more pronounced in the high-top model than in the low-top model [larger by about a factor of 2 at $10 \mathrm{hPa}$ and between a factor of 2 and 3 at $100 \mathrm{hPa}$ (Fig. 2, upper right)]. Close to the surface (Fig. 2, bottom right), the high-top model minus the low-top model difference is almost entirely due to the anomaly of the high-top model. Figure 2 shows that in autumn and in the early part of the winter, the response of the two models is in closer agreement than in late winter and early spring (February and March). A more subtle difference is that the temperature anomaly in the low-top model lacks the time-pressure vertical tilt in the sponge zone (30-10 $\mathrm{hPa}$ ). This is manifested in the large negative difference in zonal mean temperature in March at $10 \mathrm{hPa}$ (Fig. 2, bottom left). The sustained zonal mean zonal wind difference (Fig. 2, bottom right; about $1 \mathrm{~m} \mathrm{~s}^{-1}$ ) indicates that the zonal mean anomalies are weaker throughout the troposphere in February and March in the low-top model.

To further characterize the difference in the response to ENSO events in the Northern Hemisphere winter stratosphere, the ENSO anomaly in the monthly-mean geopotential height at $50 \mathrm{hPa}$ is shown in Fig. 3 for December-February for the high-top and low-top models and ERA-40. In the high-top model, the Arctic polar geopotential height anomaly evolves from being negative in December to being positive in February, behavior that is consistent with the previously reported changes in the zonal mean temperature and zonal wind. A similar evolution occurs also for the low-top model. However, in the low-top model, the positive geopotential height anomaly remains confined over Canada, hence the anomaly pattern is more wave-like. This latter behavior is consistent with the limited anomaly in zonal mean in the low-top model (Figs. 1 and 2). Conversely, in February, a more annular structure characterizes the geopotential height anomaly associated with ENSO in the high-top model. A significant negative anomaly over northern Europe is indeed present only in the hightop model. The less zonal (or annular) response of the low-top model in February is found also in the temperature (not shown here, whereas the results for the high-top model and ERA-40 are shown in Manzini et al. 2006). The large positive anomaly evolving toward the North Pole from December to February is also found for the ERA-40 data (Fig. 3, lower panels). However, some caution must be taken into account in the interpretation of the ENSO anomaly from the reanalysis because two of the winters (1982/83 and 1991/92) included in the composite follow volcanic eruptions, which occurred in the Northern Hemisphere. Therefore, the ENSO anomaly could be distorted in ERA-40, given that the effects of volcanic eruptions should be a cooling and strengthening of the vortex (negative geopotential height anomaly over the North Pole; Labitzke and van Loon 1989). 

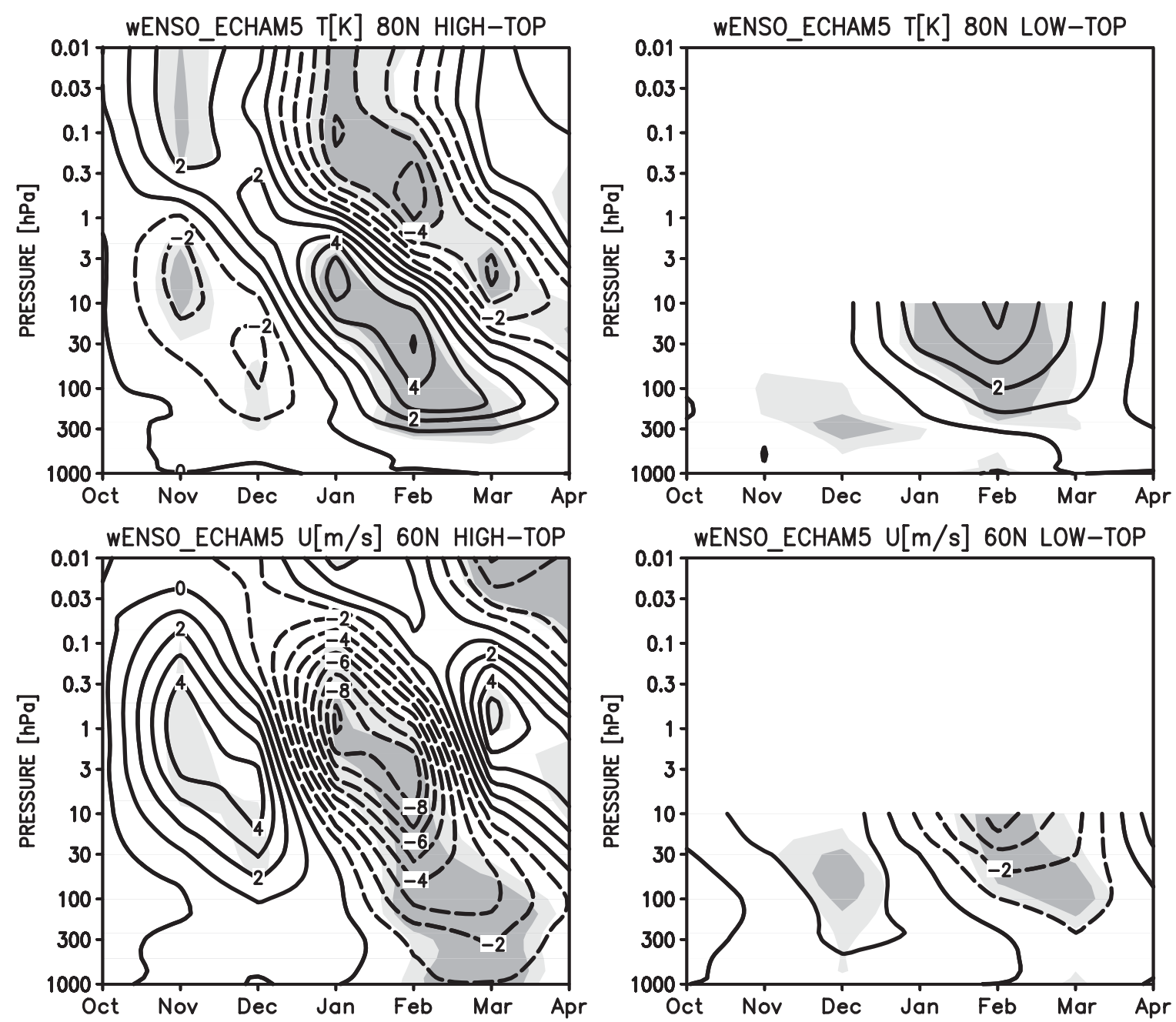

FIG. 1. October-April monthly zonal mean ENSO anomaly. (top) Temperature (contour: $1 \mathrm{~K}$ ) at $80^{\circ} \mathrm{N}$ for the (left) high-top and (right) low-top models. (bottom) Zonal wind (contour: $1 \mathrm{~m} \mathrm{~s}^{-1}$ ) at $60^{\circ} \mathrm{N}$ for the (left) high-top and (right) low-top models. Light and dark shades indicate statistical significance at the $95 \%$ and $99 \%$ levels, respectively. (Left panels reprinted from Manzini et al. 2006).

The results of Manzini et al. (2006) and the additional ones reported here for both models are broadly consistent with the finding of Taguchi and Hartmann (2006) about increased occurrence of stratospheric sudden warmings for ENSO events in their model, because a weaker polar vortex is suggestive of the occurrence of sudden stratospheric warmings. However, Manzini et al. (2006) did not explicitly look at the occurrence of these large-scale perturbations of the polar stratosphere. In the current context of understanding the high-top minus low-top model differences, it is of interest to evaluate daily variations in zonal mean temperature and zonal wind for both ensembles of simulations.

Figure 4 shows the daily evolution (from July to June) of the climatological mean (calculated for each calendar day) zonal mean zonal wind (black curve) for the high-top and low-top models at $60^{\circ} \mathrm{N}$ and at 10 (left) and $70 \mathrm{hPa}$ (right), respectively. The dark gray envelopes indicate \pm 1 total standard deviation (total meaning from the 9member $\times 20$-yr time series, therefore including internal variability). The light gray envelopes are individual maxima and minima from all daily time series (180 yr). The mean behavior of the daily zonal mean zonal wind is comparable in the two models. As reported in section 2, this is expected because the sponge in the low-top model is designed to produce a reasonably realistic mean state in the lower stratosphere. The mean zonal wind is only slightly stronger in the winter, fall, and spring seasons in the low-top model. For instance, by mid-April the vortex in the high-top model has collapsed on average, whereas the zonal mean zonal winds are still positive (albeit weak) at the beginning of May in the low-top model. 

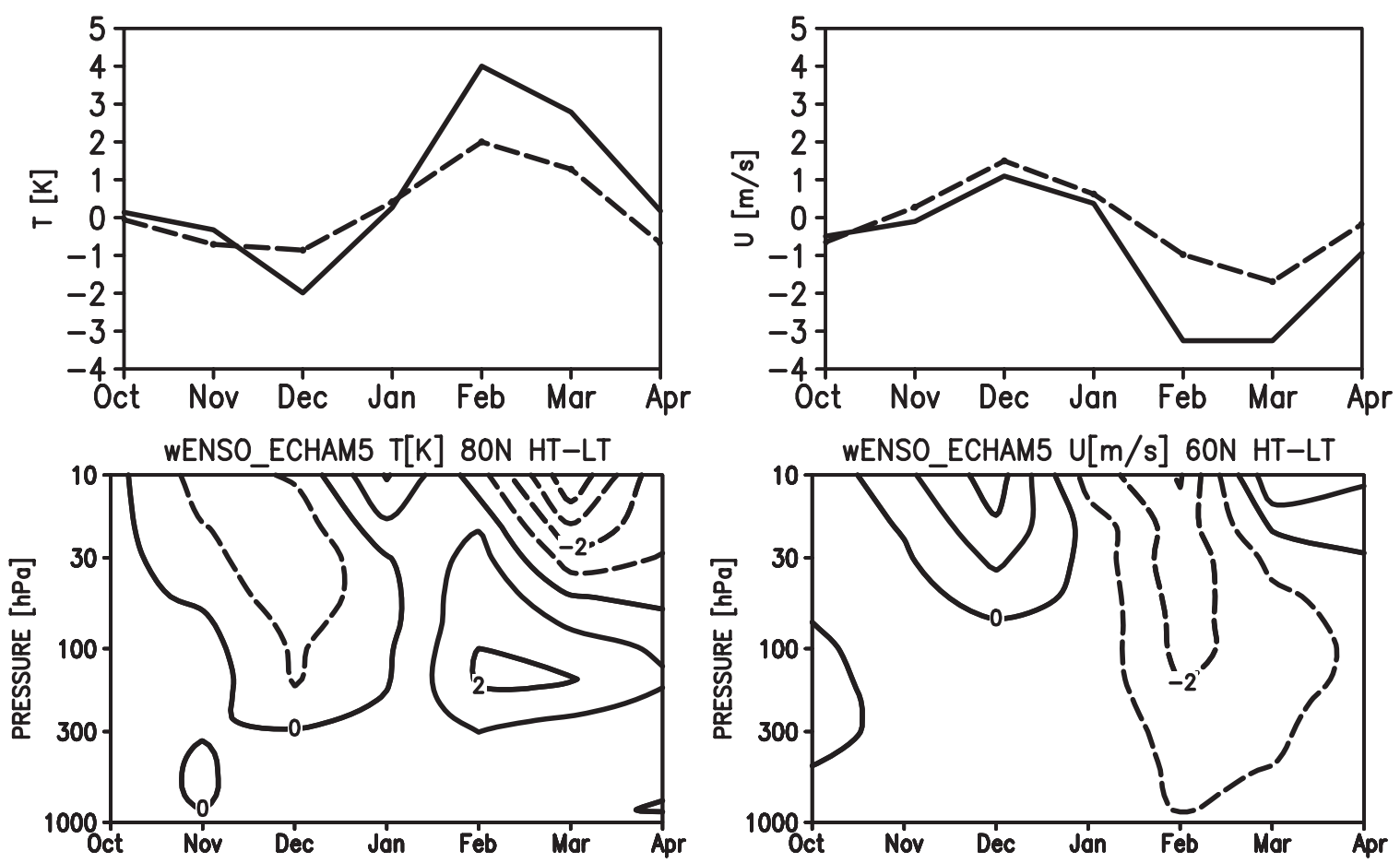

FIG. 2. (upper) October-April monthly zonal mean ENSO anomaly at $100 \mathrm{hPa}$ for the high-top (solid curve) and low-top models (dashed curve). (left) Temperature $(\mathrm{K})$ at $80^{\circ} \mathrm{N}$. (right) Zonal wind $\left(\mathrm{m} \mathrm{s}^{-1}\right.$ ) at $60^{\circ} \mathrm{N}$. (lower) High-top minus low-top model difference of the ENSO anomaly in the (left) zonal mean temperature (contour: $1 \mathrm{~K}$ ) at $80^{\circ} \mathrm{N}$ and (right) zonal mean zonal wind (contour: $1 \mathrm{~m} \mathrm{~s}^{-1}$ ) at $60^{\circ} \mathrm{N}$ from October to April, from the surface to $10 \mathrm{hPa}$.

The daily variability is instead rather different in the two models. In the high-top model, there are clearly a number of major sudden stratospheric warmings (negative zonal winds at $60^{\circ} \mathrm{N}$ and $10 \mathrm{hPa}$ ) throughout the winter season and the early spring (top panel). Major sudden stratospheric warmings are instead virtually absent in the low-top model (bottom panel)—obviously a deficiency. Moreover, the difference in the range of variability is due to not only a lack of vortex breakdowns but also to a reduced range of strong vortex episodes (the occurrence of zonal winds between 40 and 60 $\mathrm{ms}^{-1}$ ). The difference in the standard deviation between the high-top and low-top models is statistically significant from September to May ( $F$ test).

The right panels in Fig. 4 show that also at $70 \mathrm{hPa}$, just below the sponge layer of the low-top model, stratospheric variability is reduced in the low-top model. The difference in the standard deviation (up to a factor 2) is statistically significant from September to May $(F$ test).

To quantify their occurrence, statistics of major sudden stratospheric warmings in the high-top model are shown in Fig. 5. Namely, Fig. 5 shows the frequency of occurrence of sudden stratospheric warmings events per year in the extended winter season (from November to March) stratified by calendar month. The criteria used for defining a major warming event is consistent with the standard definition (Labitzke 1981): the warming occurs if the $10-\mathrm{hPa}$ meridional zonal mean temperature gradient between the North Pole and $60^{\circ} \mathrm{N}$ is positive and the zonal mean zonal wind at $10 \mathrm{hPa}$ and $60^{\circ} \mathrm{N}$ becomes easterly, for at least 4 days. Final warmings are removed from the statistics. Black numbers in Fig. 5 represent the number of members in the ensemble reporting a given frequency of occurrence of warming events per year (the number of events for each month of the ensemble member time series divided by $20 \mathrm{yr}$ ); the black diamond is the average frequency (the total number of events for the 9 members divided by $180 \mathrm{yr}$ ). For each ensemble member, the statistics are unreliable (large spread), because the number of the years is small (20 yr). Clearly, here are some ensembles members that are outliers, a consequence of the large internal variability of the winter stratosphere. The results in Fig. 5 can be compared with those of Charlton et al. (2007), their Fig. 3. Note that the model data for MAECHAM in Charlton et al. (2007) are from the high-top model of this work (i.e., the MAECHAM5 model). Indeed, the last 20-yr simulation of the 30-yr simulation considered in Charlton et al. (2007) is one of the members depicted in Fig. 5. In their Fig. 3, Charlton et al. (2007) compared 
wENSO $50 \mathrm{hPa} Z[\mathrm{~m}] \mathrm{m}=$ Dec HIGH-TOP

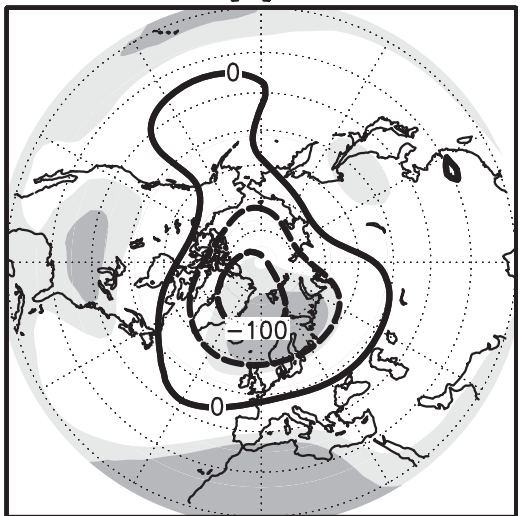

WENSO $50 \mathrm{hPa} Z[\mathrm{~m}] \mathrm{m}=$ Dec LOW-TOP

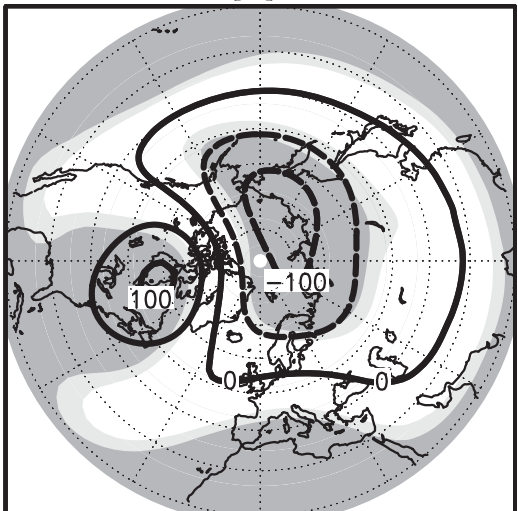

wENSO $50 \mathrm{hPo} Z[\mathrm{~m}] \mathrm{m}=$ Dec ERA40

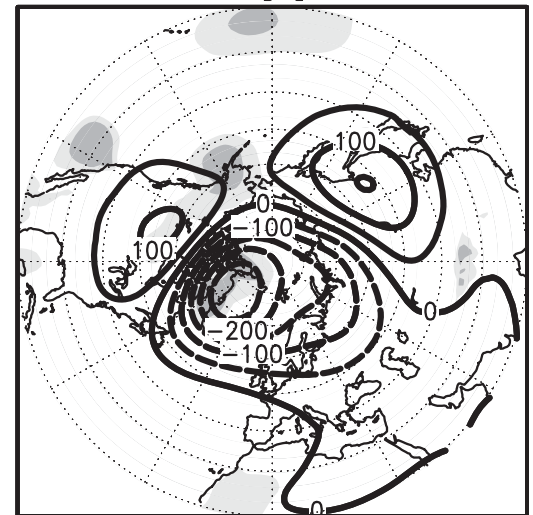

wENSO $50 \mathrm{hPa} Z[\mathrm{~m}] \mathrm{m}=\mathrm{Jan}$ HIGH-TOP

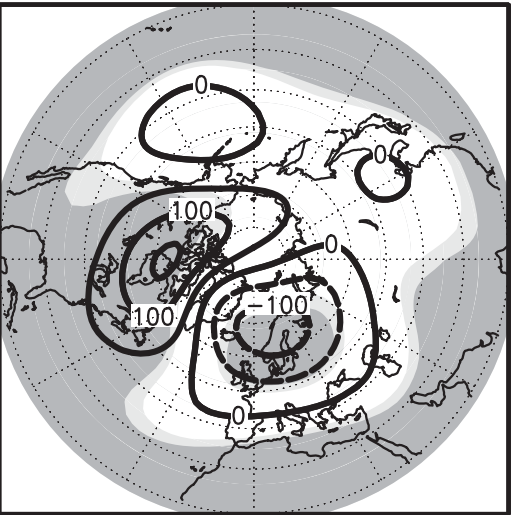

WENSO $50 \mathrm{hPa} Z[\mathrm{~m}] \mathrm{m}=$ Jan LOW-TOP

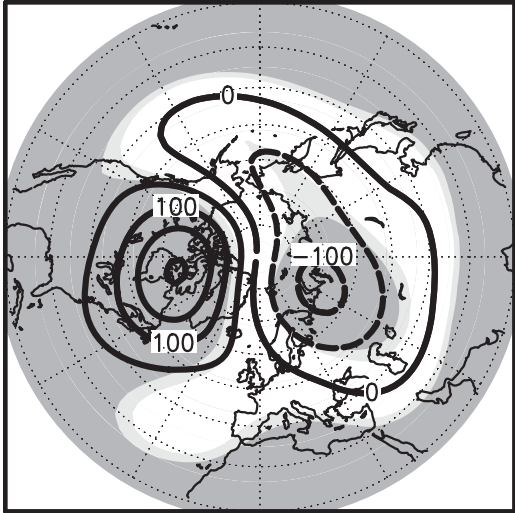

wENSO $50 \mathrm{hPa} Z[\mathrm{~m}] \mathrm{m}=\mathrm{Jan}$ ERA40

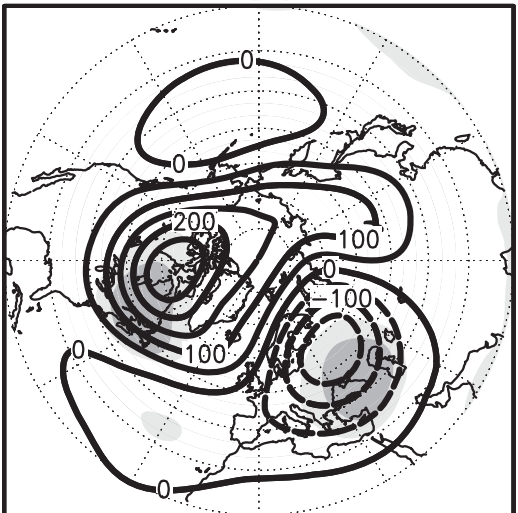

wENSO $50 \mathrm{hPa} Z[\mathrm{~m}] \mathrm{m}=\mathrm{Feb}$ HIGH-TOP

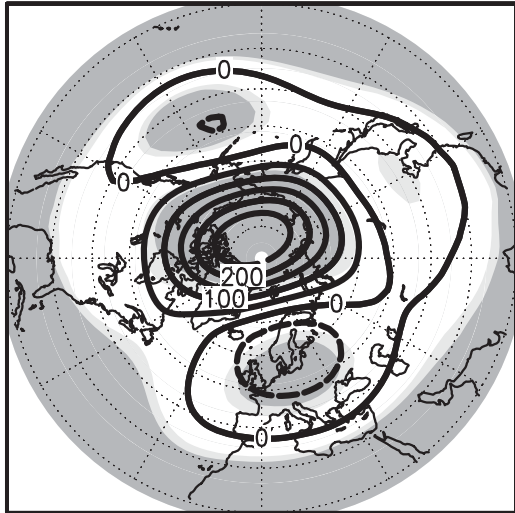

wENSO $50 \mathrm{hPa} Z[\mathrm{~m}] \mathrm{m}=\mathrm{Feb}$ LOW-TOP

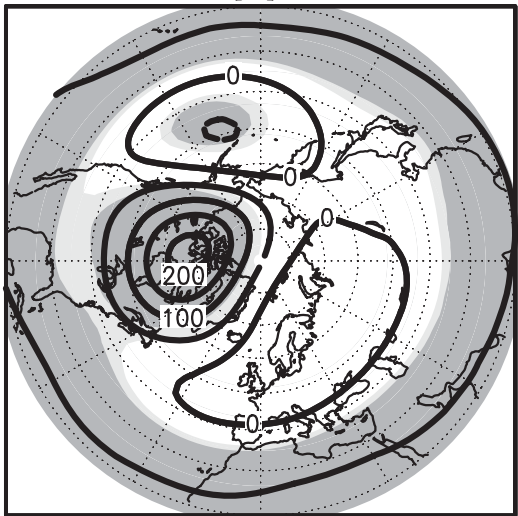

wENSO $50 \mathrm{hPa} Z[\mathrm{~m}] \mathrm{m}=\mathrm{Feb}$ ERA40

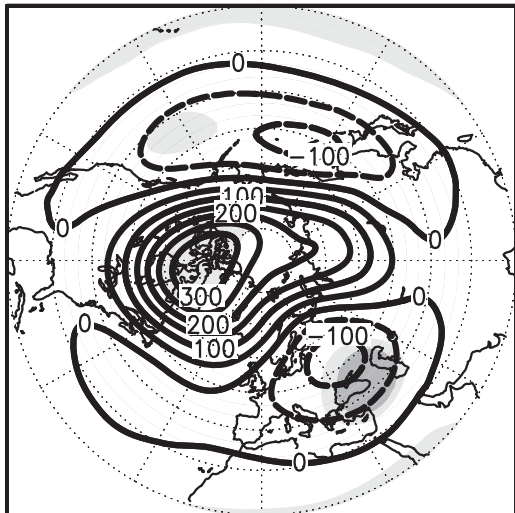

FIG. 3. (left) December, (middle) January, and (right) February ENSO anomaly in geopotential height (contour: $50 \mathrm{~m}$ ) at $50 \mathrm{hPa}$ for (top) high-top model, (middle) low-top model, and (bottom) ERA-40 data. Light and dark shades indicate statistical significance at the $95 \%$ and $99 \%$ levels, respectively.

the sudden stratospheric warmings, in frequency of events per year stratified by month, of a number of models to the NCEP-NCAR reanalysis. Concerning the MAECHAM5 model, their figure shows a possible overestimation of the frequency of events in November and underestimation from January to March with respect to the frequency deduced from the National Centers for Environmental Prediction-National Center for Atmospheric Research (NCEP-NCAR) reanalysis. Figure 5 demonstrates that by simply considering more cases, the MAECHAM5 sudden stratospheric warming climatology is improved with respect to the one shown in Charlton et al. (2007), because of the reduction in frequency in November and the increase in March, when considering the total number of $180 \mathrm{yr}$. Moreover, all the Charlton et al. (2007) observed frequencies 

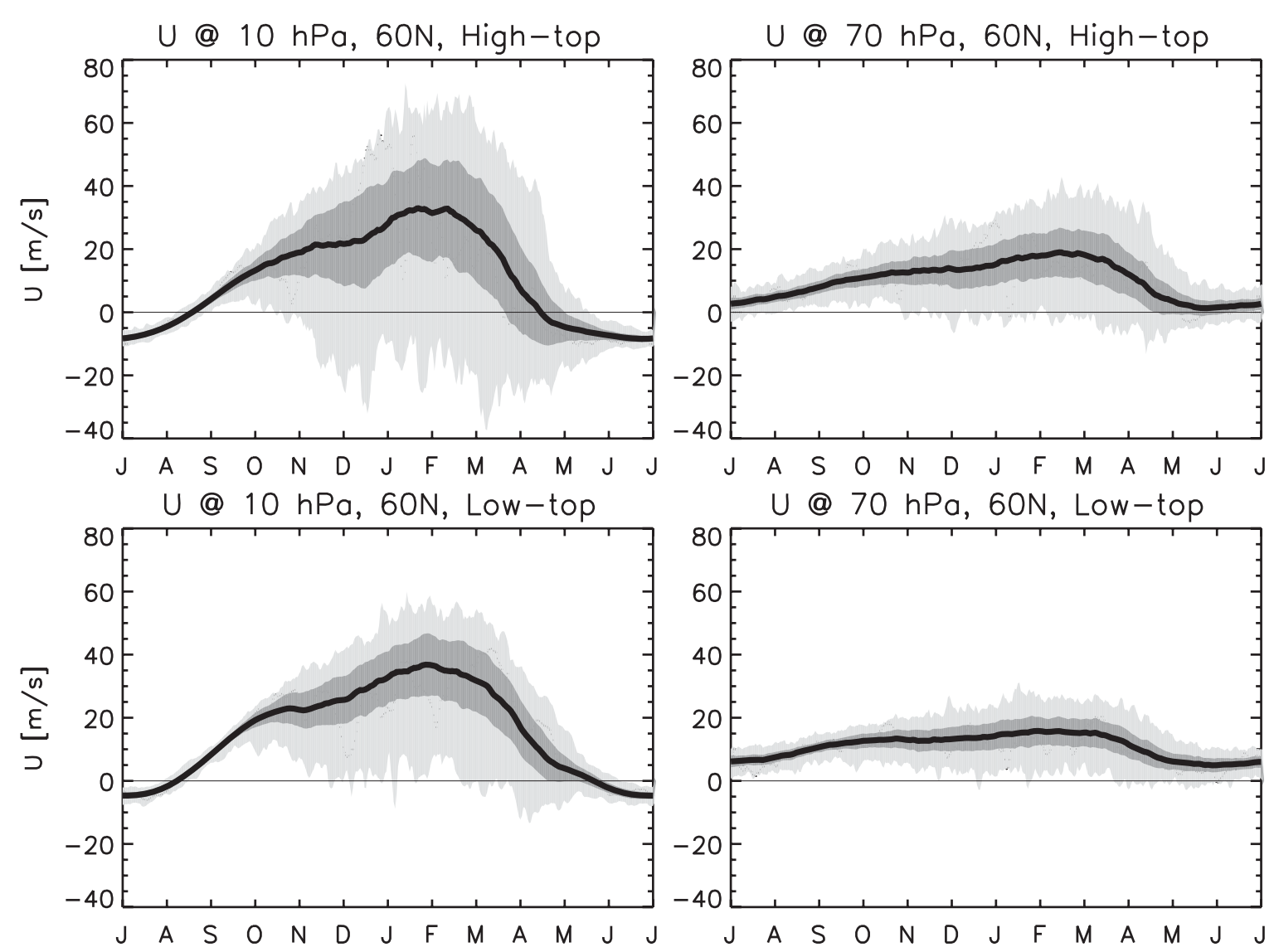

FIG. 4. July to June daily zonal mean zonal wind $\left(\mathrm{m} \mathrm{s}^{-1}\right)$ at $60^{\circ} \mathrm{N}$ and (left) $10 \mathrm{hPa}$ and (right) $70 \mathrm{hPa}$ for the (top) high-top and (bottom) low-top models. Black curve: climatological average $(20 \mathrm{yr} \times 9$-member set $)$ daily time series. Dark gray curve: daily time series of \pm 1 total standard deviation (for the $20 \mathrm{yr} \times 9$-member set). Light gray curve: daily maxima and minima values.

(based on $45 \mathrm{yr}$, which is about twice of each of our members) fall into the MAECHAM5 range results, indicating care in not over interpreting the observations. Indeed, the frequency for the full winter (not stratified by month) is probably very realistic for the MAECHAM5 model. Therefore, we can conclude from comparing with observations that the stratospheric variability of the high-top model is in good agreement with observations and, most importantly for our case here, not overestimated.

Once it has been established that the variability in the high-top model in the stratosphere is broadly realistic, it is of interest to show the daily time series of the ensemble mean zonal mean zonal wind stratified by ENSO. In the case of the high-top model, the daily time series (from July to June) of the ensemble mean zonal mean zonal wind $\left(10 \mathrm{hPa}\right.$ and $\left.60^{\circ} \mathrm{N}\right)$ stratified by ENSO is depicted in Fig. 6 (thick red curves). Each individual ensemble member time series is also shown in Fig. 6 (thin red curves). The black curves and the gray envelopes are from Fig. 4. From visual inspection of the panels of Fig. 6, it is evident that the ensemble mean time series for the 1997/98 winter is dominated by an external forcing. From January to April, the 1997/98 polar vortex is weaker: the ensemble mean zonal wind is clearly smaller than the climatological mean. This is because major sudden stratospheric warming events occur for a number of the individual members in 1997/ 98 (thin red curves). A negative deviation of the ensemble mean zonal mean zonal wind time series to the climatology is also found for 1982/83 in January and February (note that there are no volcanic effects in the model). Also in this case, a number of major warmings are seen from Fig. 6. During both winters, the ENSO events are known to have been strong. The ensemble mean time series for the other two cases (weaker ENSO events) considered are, instead, close to the climatology. The 1986/87 winter has a few members disturbed in December and thereafter in March, a behavior sometimes observed, whereas the 1991/92 winter is slightly more disturbed in February and March. The results for the two strong ENSO events demonstrate that the polar 


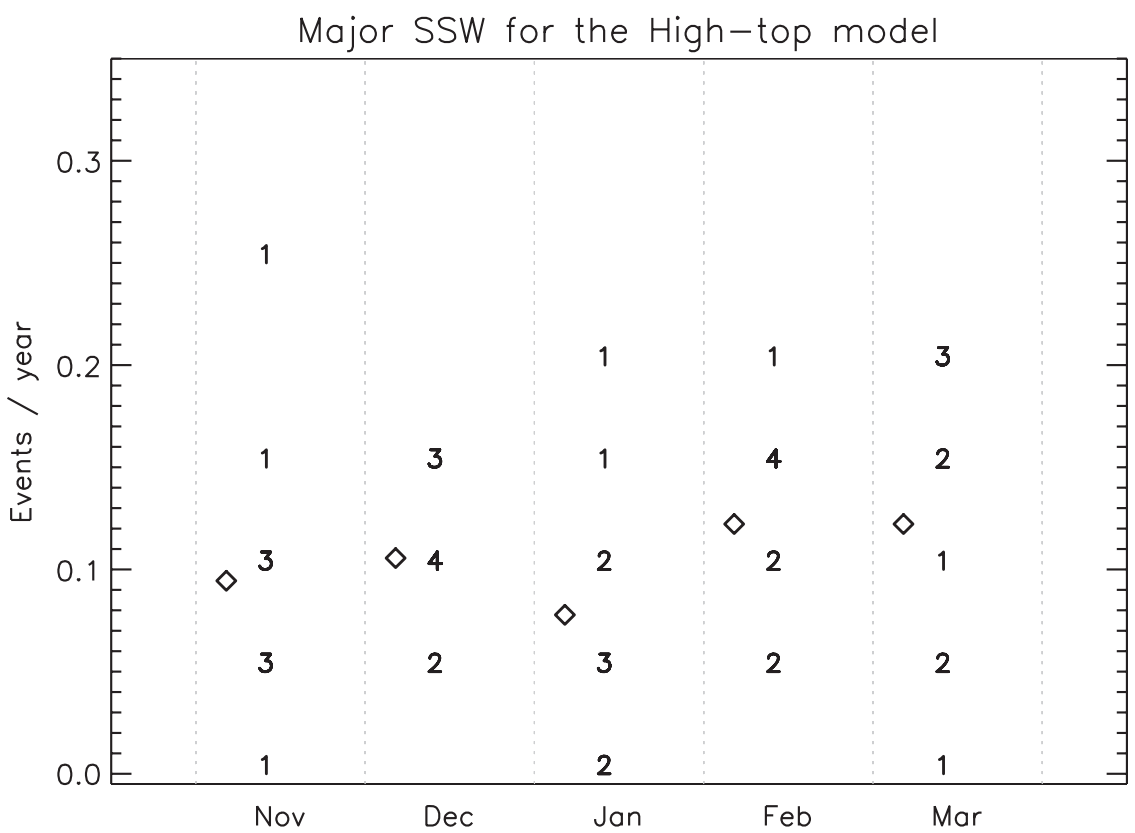

FIG. 5. Major sudden stratospheric warming statistics stratified by month, November-March. Black numbers: number of ensemble members with a given frequency of occurrence of sudden stratospheric warming events. Diamonds: average frequency (total number of events for the 20 yr $\times 9$-member set divided by $180 \mathrm{yr}$ ).

warming occurring in the stratosphere of the high-top model is associated with sudden stratospheric warmings. Our results for the high-top model are, therefore, consistent with those of Taguchi and Hartmann (2006).

The corresponding results for the four ENSO winters for the low-top model are shown in Fig. 7. As expected from Fig. 4, there are no major warmings for the low-top model. Nevertheless, the subset of the ENSO winters shows a tendency to be more disturbed (pulses of weaker winds), especially for the 1982/83 and 1997/98 winters.

\section{b. ENSO signal at the surface in late winter/early spring}

In this section, we concentrate on late winter and early spring because Figs. 1 and 2 suggest that this is the period when the downward influence of the stratosphere on the troposphere is the strongest.

Figure 8 shows the ENSO anomaly in SLP for January-March from the high-top and the low-top models and for the ERA-40 reanalysis. The negative anomaly that characterizes the tropical extratropical ENSO teleconnections for the Northern Pacific region in winter (Hoerling et al. 1997) is captured by both models. The ENSO anomaly in the North Pacific appears to be more persistent in time in the models, being still present in March, than for the ERA-40 data. The ENSO anomaly is not statistically significant in the North Pacific in February and March in the ERA-40 data, indicating a dominance of variability (either internal or from other sources) as well as a role for ENSO interevent variability. It is possible that both models are overemphasizing the response in the North Pacific, a result of a bias of the tropospheric part of the models or of the methodology used (analysis of ensembles of simulations with prescribed SSTs). Moreover, the simulations exclude other sources of variability and change, such as varying greenhouse gases and aerosols, volcanoes, quasi-biennial oscillation, and solar variations. Possibly, a much larger number of ENSO events would be needed to extract an ENSO signal-if any-in the North Pacific in March from observations. This aspect is beyond the scope of this investigation and is not further researched here. The experimental design used and the lack of a number of other sources of variability and change in the models are, of course, facilitating the extraction of the ENSO response, however, making the comparison with observations more ambiguous.

The interesting aspect of Fig. 8 is that the evolution of the ENSO SLP anomaly over the Arctic and the NAE region is different in the two models. For the high-top model, a positive pressure anomaly develops over the Arctic (about $8 \mathrm{hPa}$ in March) and a negative pressure anomaly over the North Atlantic and European region (about $-4 \mathrm{hPa}$ in March). All the centers of the SLP anomalies in March are statistically significant in the high-top model. This evolution is hinted at in the low-top 

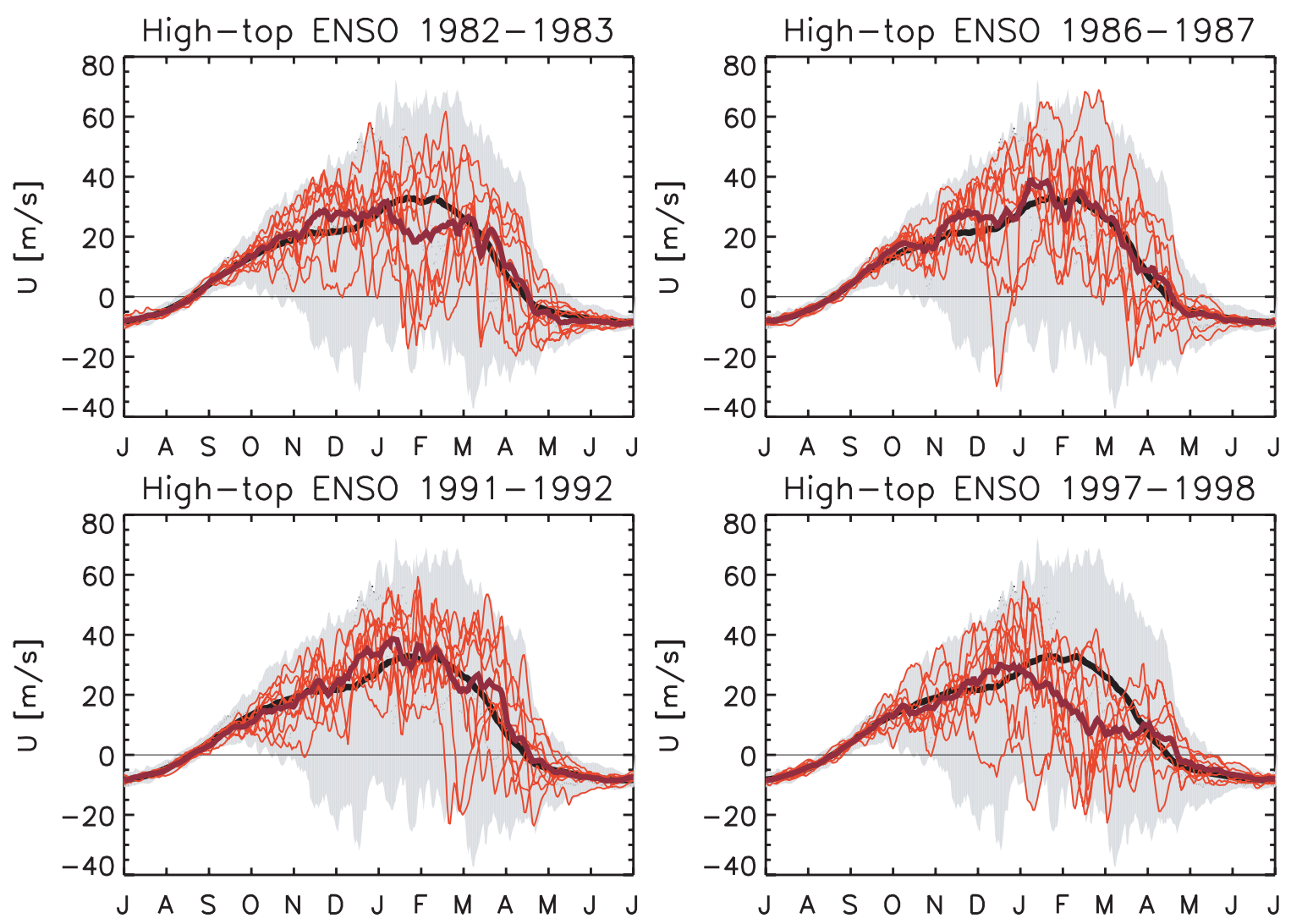

FIG. 6. July-June daily zonal mean zonal wind $\left(\mathrm{m} \mathrm{s}^{-1}\right)$ at $60^{\circ} \mathrm{N}$ and $10 \mathrm{hPa}$ for the high-top model. Dark red curves: ensemble average (9-member set) stratified by ENSO event for (top left) 1982/83, (top right) 1986/87, (bottom left) 1991/92, and (bottom right) 1997/98. Light red curves: individual members stratified by ENSO event. Black and light gray curves from Fig. 4 (top, left).

model, leading to anomalies of the same sign and analogous patterns in March, albeit much weaker (especially over the Arctic) and not significant. The ERA-40 data, although based on only four ENSO events, also show a large and significant positive anomaly over the Arctic and negative anomaly over the NAE region in March, substantiating the high-top model results. The results of the high-top model are consistent with the ENSONAE teleconnection found, for instance, in the work of Fraedrich and Müller (1992), the so-called canonical response to ENSO summarized in Brönnimann (2007).

Inspection of the SLP anomaly for the individual events (not shown) reveals that for the high-top model, the anomaly pattern over the Arctic and NAE regions is consistent among the events, whereas it is more occasional-even absent-for the low-top model. Clearly for the individual events, but also in the composite, ERA-40 is more dominated by small-scale disturbances.

Given that the model formulation in the lower atmosphere is identical in the high-top and low-top models, a role for the stratosphere in organizing the SLP anomalies in the northern late winter and early spring tropo- sphere is implied. This interpretation is consistent with the high-top minus low-top model difference in the zonal mean zonal wind anomalies in the troposphere (Fig. 2).

To further assess and quantify the role of the stratosphere on surface climate in late winter and early spring, Fig. 9 shows the FM average of the ENSO anomalies for the SLP, the 1000-hPa temperature, and the precipitation for the high-top and low-top models. Figure 9 (right panel) shows SLP and 1000-hPa temperature for the ERA-40 data and precipitation (updated from Xie and Arkin 1997). In Fig. 10 the difference (high-top model minus low-top model) for the three surface field anomalies considered is depicted. The ENSO anomaly in SLP for the FM average (Figs. 9 and 10) point, again, to the more annular (or zonal) character of the high-top model results. The SLP anomaly difference (Fig. 10) is about -1 over western Europe, +6 over the Arctic, and $-2 \mathrm{hPa}$ over the North Pacific. For both models, the statistical significance (not shown) of the centers of the SLP anomalies for the FM average is comparable to that of the two individual months (Fig. 8). In summary, the more zonal (or annular) late winter-early spring 

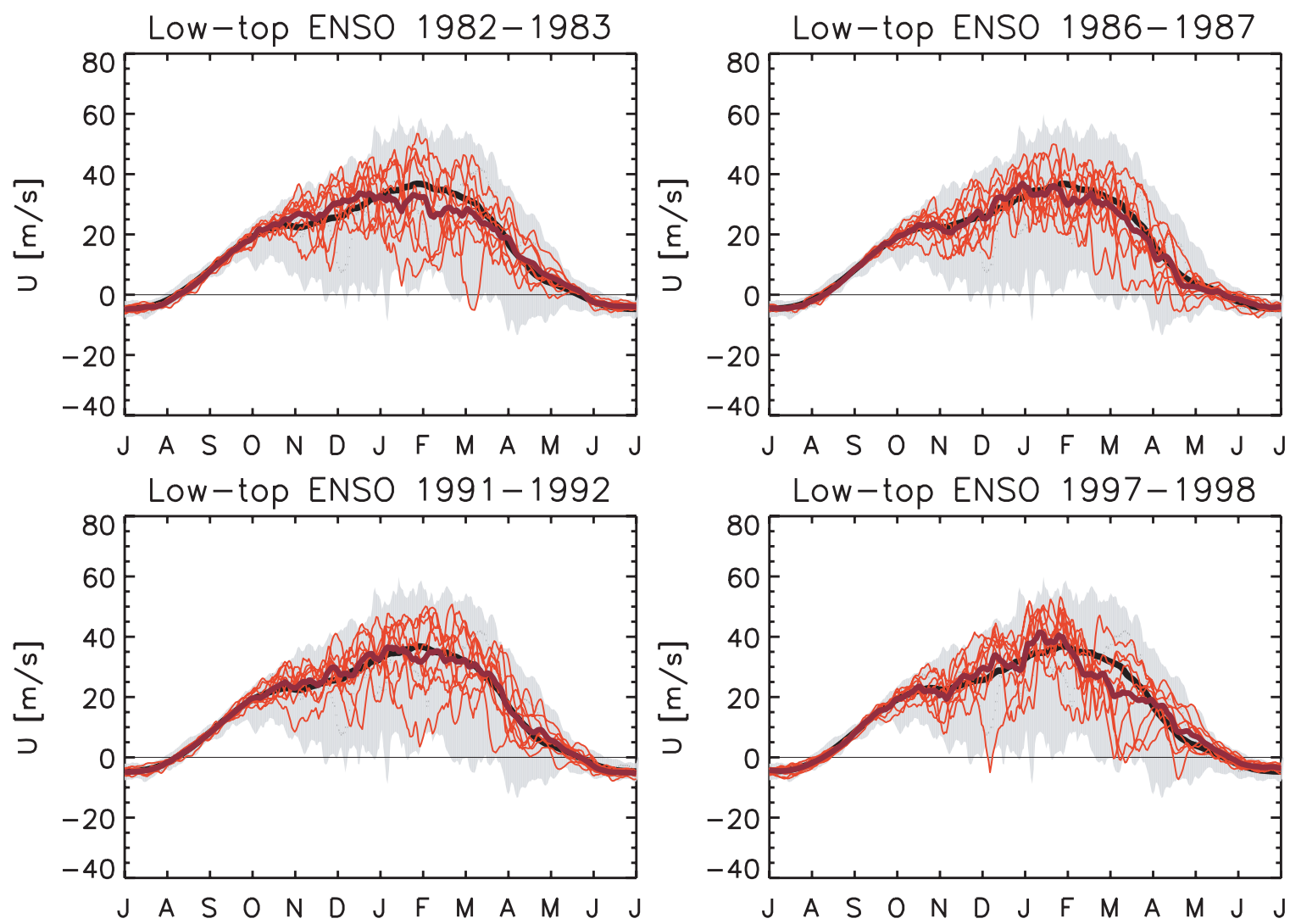

FIG. 7. Same as Fig. 6 but for the low-top model.

behavior that characterizes the response to ENSO in the high-top model reported for the lower stratosphere (Fig. 3) is also found at the surface.

At the 500-hPa level (not shown), the geopotential height ENSO respnsein the high-top model is also more annular. However, synoptic-scale anomalies are more substantial at this level, because baroclinic disturbances dominate the flow in the middle troposphere (Thompson and Wallace 1998).

Focusing on the Arctic and NAE regions, where the relative difference in the ENSO response in the two models are more substantial, Fig. 9 shows that the ENSO anomaly in the $1000-\mathrm{hPa}$ temperature for the high-top model is positive over the Arctic and negative over northern and central Europe (about $-1 \mathrm{~K}$ over Scandinavia, with statistical significance at the $95 \%$ level; not shown), and Siberia (about $-1.5 \mathrm{~K}$, with statistical significance at the $99 \%$ level; not shown). This result is consistent with the anomalies in SLP. The reported high SLP anomaly over the Arctic and low SLP anomaly over central Europe implies anomalous easterlies at the surface, hence reduction of advection of warm air from the North Atlantic Ocean to northern and central Europe. A similar pattern is also seen from the ERA-40 data (middle right, Fig. 9; not statistically significant). In the low-top model, the negative anomaly over Eurasia is weaker and confined to its northern latitudes, and the positive anomaly over the Arctic is virtually absent (not statistically significant). Figure 10 substantiates this result, showing that the temperature difference is slightly positive $(1 \mathrm{~K})$ over the Arctic and negative over northern and central Europe and Siberia. For central Europe the difference reaches $-2 \mathrm{~K}$ (Fig. 10).

For the NAE region, the ENSO anomaly in precipitation (Fig. 9) is characterized by a similar anomaly in the high-top and low-top models, showing a southward shift of the North Atlantic precipitation band associated with a displacement of the storm track toward southern Europe. This behavior also emerges for the Xie and Arkin (1997) data, but it is not statistically significant. Also in this case, the anomaly is stronger for the high-top model, about 50\% more over western and southern Europe (Fig. 10). Over western and central Europe, the precipitation anomaly is statistically significant at the $99 \%$ level for the high-top model. The precipitation anomalies are not significant in the low-top model (not shown). 

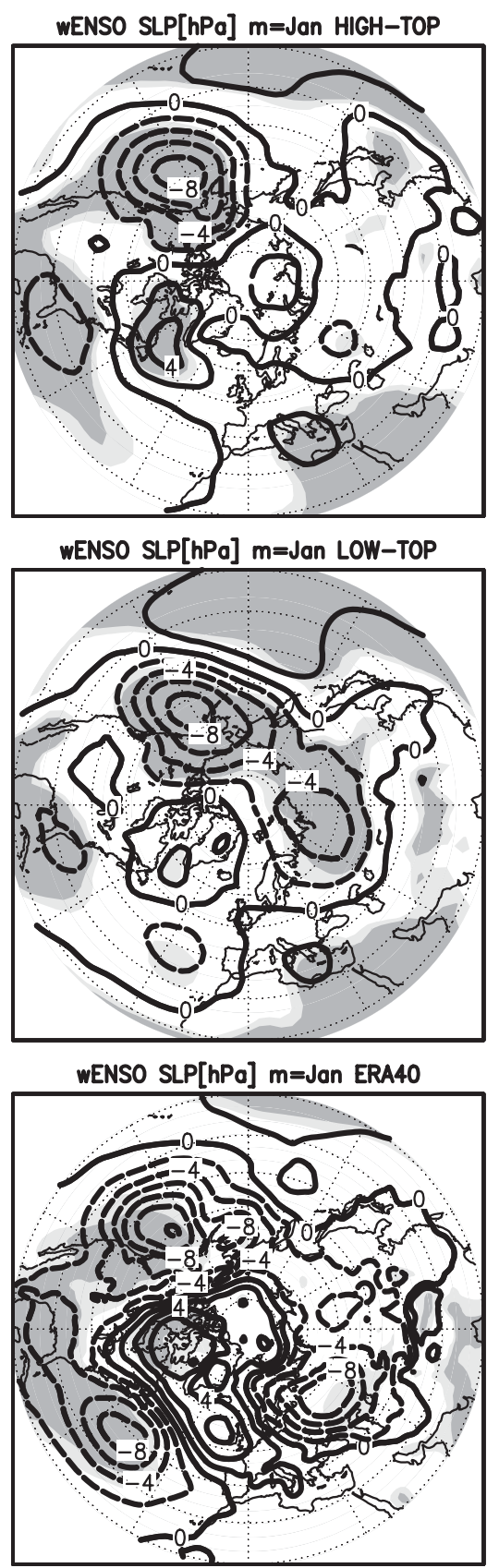
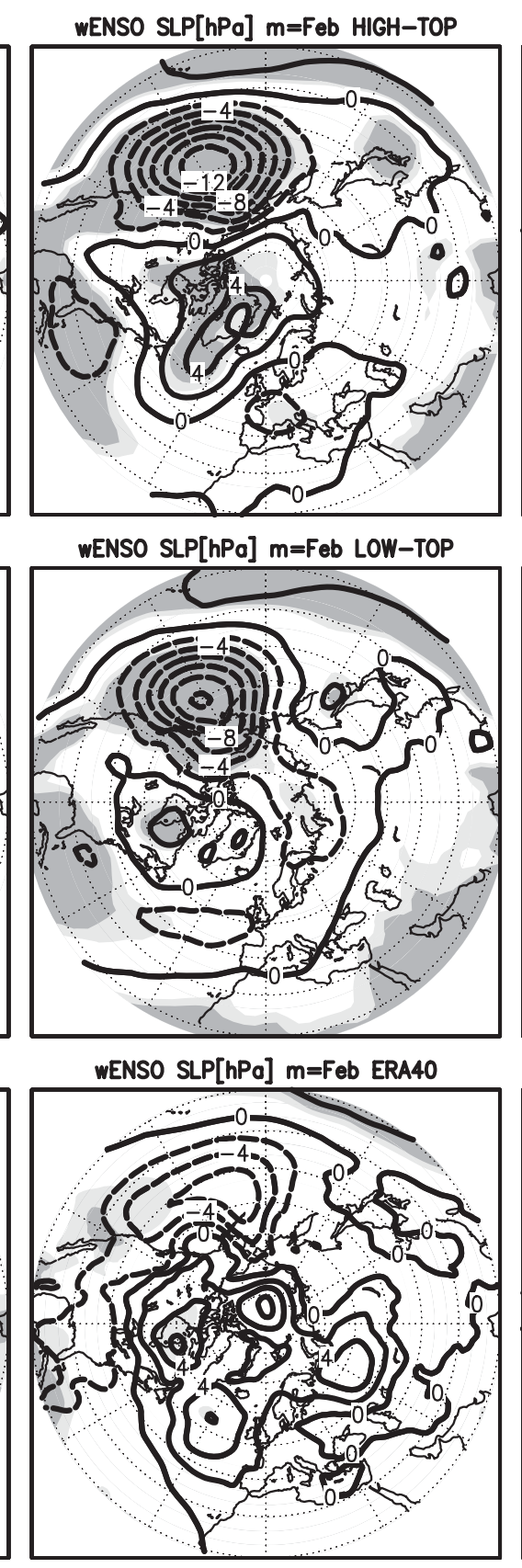

wENSO SLP[hPo] $m=$ Mar HIGH-TOP

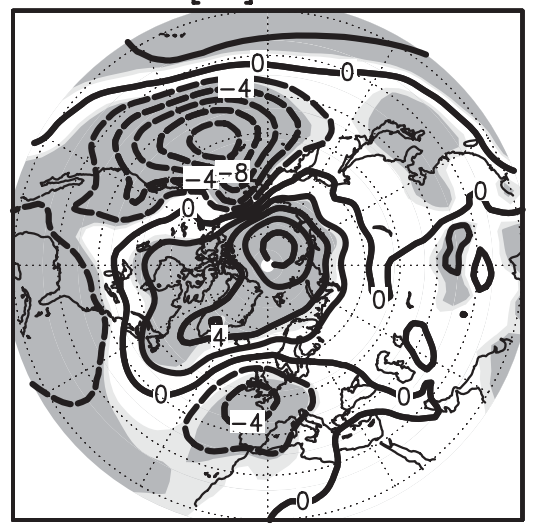

wENSO SLP[hPa] $m=$ Mar LOW-TOP

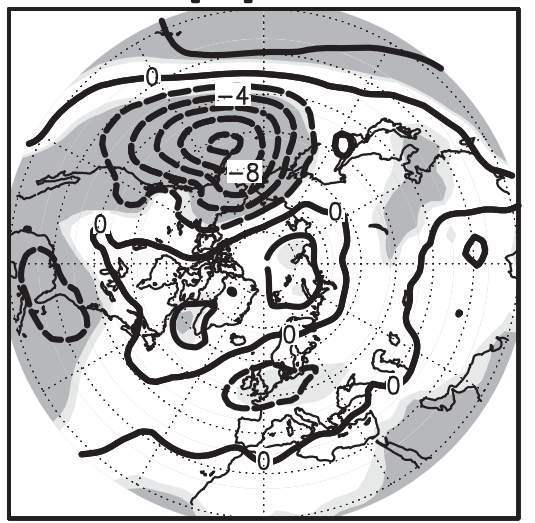

WENSO SLP[hPa] $m=$ Mar ERA40

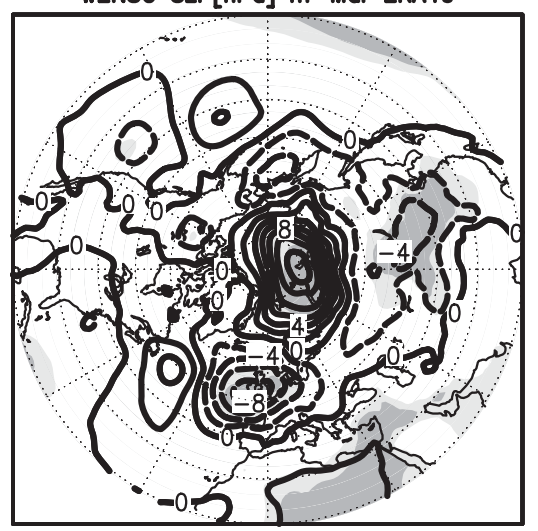

FIG. 8. (right) January, (middle) February, and (right) March ENSO anomaly in SLP (contour: 2 hPa) for (top) high-top model, (middle) low-top model, and (bottom) ERA-40 data. Light and dark shades indicate statistical significance at the $95 \%$ and $99 \%$ levels, respectively.

In summary, both models show anomalies in tropospheric surface climate over the North Atlantic and Europe that are broadly consistent with the ENSO signal in late winter over Europe, consisting of low temperatures in northern Europe, low SLP over central and western Europe, and increased precipitation over southern Europe (Fraedrich and Müller 1992; Mathieu et al. 2004). However, the strength of the response is larger and more realistic for the high-top model, pointing to a role for the stratosphere in the ENSO teleconnection over Europe.

\section{Discussion and conclusions}

The role of stratospheric variability on the winter tropospheric teleconnection between ENSO and the 

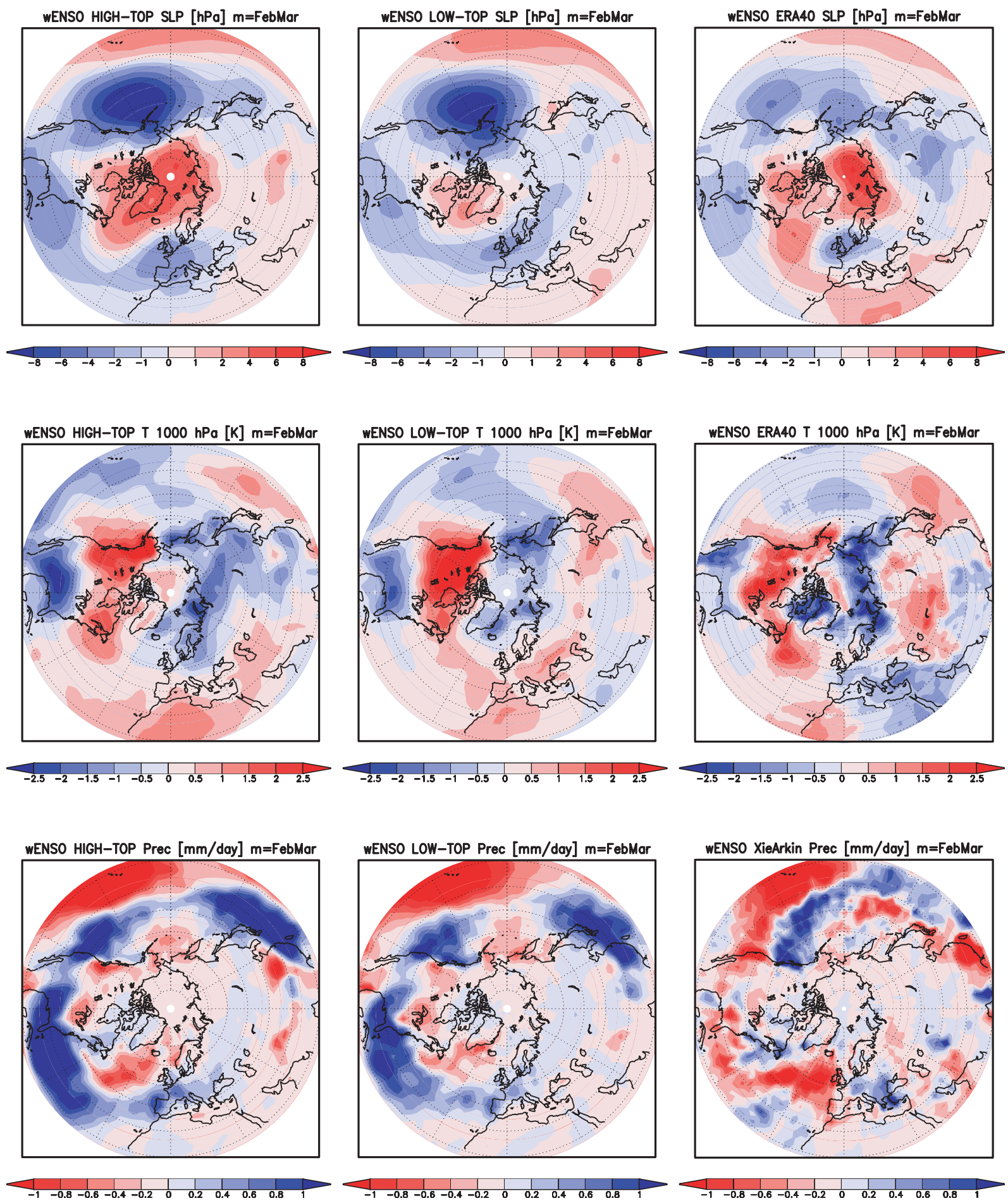

FIG. 9. FM ENSO anomaly in (top) SLP (hPa), (middle) 1000-hPa temperature (K), and (bottom) precipitation (mm day ${ }^{-1}$ ) for (left) hightop model, (middle) low-top model, and (right) ERA-40 data for SLP and 1000-hPa temperature; precipitation data from observations.

North Atlantic and European region has been investigated by analyzing results from atmospheric general circulation models with and without a well-resolved stratosphere. Results have been compared from two ensembles of nine simulations each, performed with the high-top and low-top versions of the same atmospheric general circulation model, respectively. Each simulation is 20-yr long and is forced by prescribed observed sea 
(HT - LT) SLP [hPa] m=FebMar

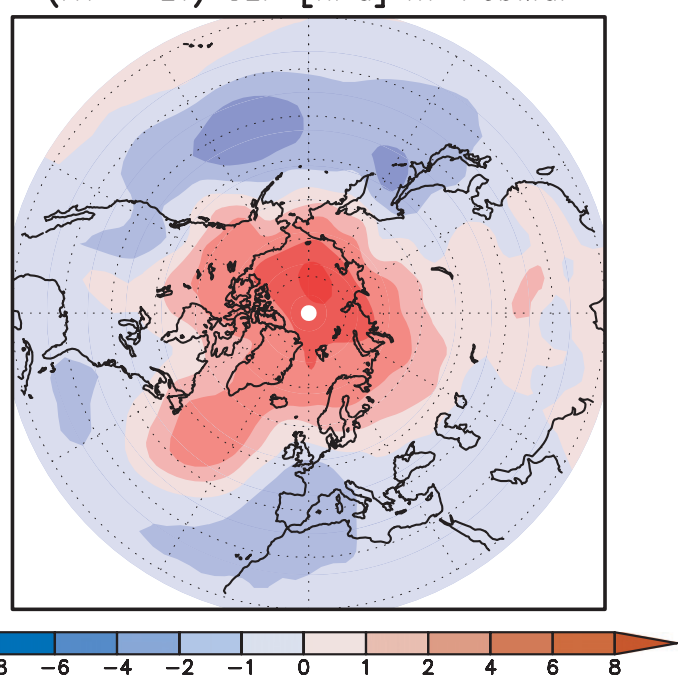

(HT-LT) T $1000 \mathrm{hPa}[\mathrm{K}] \mathrm{m}=\mathrm{FebMar}$

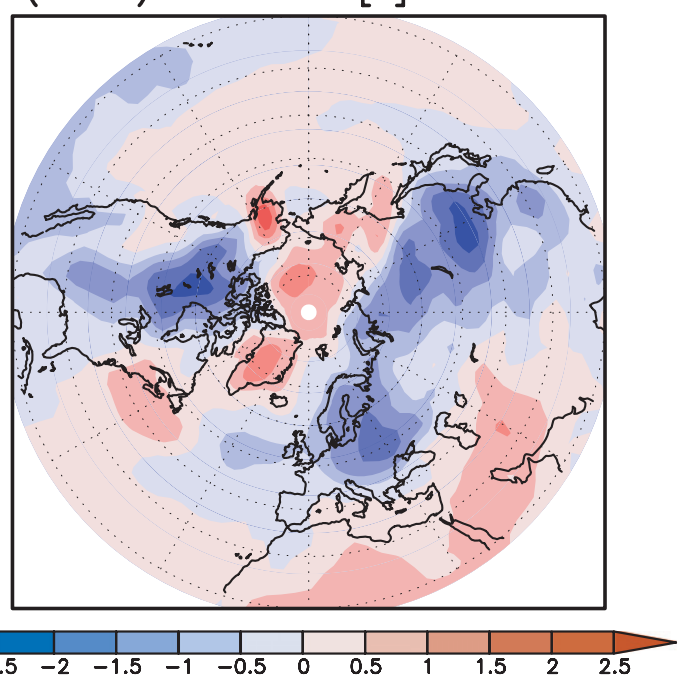

(HT - LT) Prec [mm/day] $m=F e b M a r$

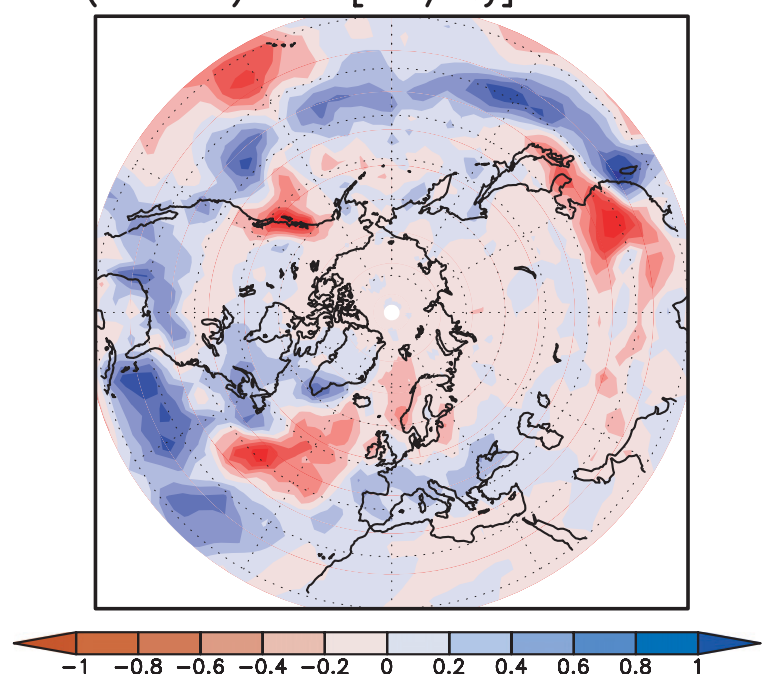

surface temperatures from 1980 to 1999 . Next are the main results obtained in this work.

1) The ENSO anomaly in the northern polar lower stratosphere, consisting of a polar warming of a few degrees and a polar vortex weakening in late winter, is also reproduced in the ensemble that does not include a well-resolved stratosphere, the low-top model (Fig. 1). This result is consistent with the interpretation that the ENSO perturbation of the northern polar stratosphere is realized as an anomalously large planetary wave forcing emerging from the troposphere (Manzini et al. 2006), a dynamical process common to both the high-top and low-top models. However, there are subtle differences in how the ENSO anomaly is realized already in the lower stratosphere. In February and March, the ENSO anomalies in zonal mean temperature and zonal mean zonal wind are reduced to about half in size in the low-top model at $100 \mathrm{hPa}$ (Fig. 2). In the low-top model, the result for the ENSO zonal mean anomalies is consistent with a less annular anomaly in geopotential height at $50 \mathrm{hPa}$ during winter (Fig. 3 ). The sustained zonal mean zonal wind difference (Fig. 2) indicates that the zonal mean anomalies are weaker throughout the troposphere in February and March in the low-top model.

2) The virtual lack of sudden stratospheric warmings in the low-top model (Figs. 4 and 7) clearly demonstrates that planetary waves do not grow enough in the stratosphere of the low-top model. Planetary waves are, indeed, damped in the sponge layer of the low-top model. Stratospheric variability measured as the occurrence of major stratospheric warming events has been found to be realistic in the high-top model (Figs. 4-6). Because the middle and high stratospheres are missing in the low-top model and planetary waves are subjected to artificial damping in the lower stratosphere of the low top model, the picture that emerge is that wave-mean-flow interaction does not fully occur, and the planetary wave perturbations retain their wave-like character in the low-top model.

3) The inspection of the SLP anomalies reveals that the more annular (or zonal) character of the ENSO anomaly found for the high-top model in the lower stratosphere in February (Fig. 3) is also manifested at the surface in March (Fig. 8). The ENSO SLP

FIG. 10. High-top minus low-top model difference in the FM ENSO anomaly for (top) SLP (hPa), (middle) 1000-hPa temperature $(\mathrm{K})$, and (bottom) precipitation $\left(\mathrm{mm} \mathrm{day}^{-1}\right)$. 
anomaly in the high-top model is characterized by a large-scale and coherent pattern, with higher pressures over the Arctic and lower pressures over western Europe and the North Pacific. In relative terms, the two models differ most in the Arctic and NAE regions. Focusing on the NAE region, the high-top minus low-top model difference in the ENSO SLP anomalies is consistent with cooling in the northern and central Europe region (difference up to $-2 \mathrm{~K}$ ) and a southward shift and increase (of about $50 \%$ ) of the precipitation band associated with the North Atlantic storm track (Fig. 9 and 10).

The major difference in the dynamical behavior of the two models is the virtual lack of sudden stratospheric warming events in the low-top model. It is, therefore, concluded that (i) the occurrence of these extreme stratospheric events affects the ENSO-NAE teleconnection; and (ii) the missing process in the low-top model is wave-mean-flow interaction due to the occurrence of sudden stratospheric warming events in the middle stratosphere.

Although other factors cannot be ruled out and may also enhance the sensitivity of the ENSO response in the Arctic and NAE regions in the troposphere, the numerical experiments carried out have clearly extracted a sensitivity of the winter tropospheric ENSO-NAE teleconnection to the modeling of the stratosphere.

Limited modeling of the stratosphere includes all the features of a model associated with a poor representation of the stratosphere, including not only the location of the model top and the vertical resolution in the model stratosphere but also the vertical resolution in the tropopause region, which is the dissipation (and its possible spurious effects) close to the upper boundary of the lowtop model. Although the finer vertical resolution of the high-top model already above $500 \mathrm{hPa}$ may also play a role in the downward transfer of a stratospheric signal, this difference is not the main cause for the reported difference at the surface between the two models, because we have shown that the stratospheric signal (wavemean-flow interaction) itself is severely misrepresented in the low-top model.

The essence of the high-top minus low-top model difference is a more annular (or zonal) pattern of the anomaly in SLP, relatively larger over the Arctic and the NAE regions. Our interpretation of the role of sudden stratospheric warming events in the ENSO-NAE teleconnection is, therefore, consistent with the observational evidence that sudden stratospheric warming events play a role in giving rise to persistent Arctic Oscillation anomalies at the surface (Baldwin and Dunkerton 2001). Beyond showing the role of sudden stratospheric warming events, the mechanism of transmission of the stratospheretroposphere connection is not addressed here. However, we would like to note that the high-top minus low-top model difference in the sea level pressure anomaly is consistent with a mechanism connecting the stratosphere to the surface via changes in the mass circulation.

We have shown that the variability of the lower stratosphere plays a role in the manifestation of the ENSONAE teleconnection, presumably and most importantly for the strong ENSO events that may affect also the North Atlantic and the European region. These results show the importance of including the representation of a well-resolved stratosphere in the modeling of climate variability and prediction for the North Atlantic-European region. However, given the large internal variability of the winter stratosphere, including a proper modeling of the stratosphere may not lead to a direct improvement in predictability, but rather to an improvement of the modeling of the troposphere-stratosphere system by removing a model bias, in this case the systematic underdetermination of stratospheric variability.

In summary, the ENSO-NAE teleconnection can be facilitated by sudden stratospheric warming events. These extreme stratospheric events are more frequent during ENSO. Therefore, they may provide a positive dynamical feedback within the troposphere-stratosphere system. This interpretation is consistent with the chain of effects characterizing the troposphere-stratospheretroposphere events that has been proposed by Reichler et al. (2005).

The methodology used (ensembles) has allowed for the extraction of the ENSO tropospheric teleconnections and the difference in the ENSO anomaly in SLP, 1000$\mathrm{hPa}$ temperature, and precipitation between the high-top and the low-top models. However, it is difficult to compare quantitatively the ensemble results with observations (or reanalysis data) because of the high level of internal variability present in the observed record. In addition, the use of prescribed sea surface temperature may have "overpredicted" the surface response to SSTs (van Oldenborgh 2005). Therefore, it would be of interest to investigate if a similar sensitivity in the ENSO response to the modeling of the stratosphere is also obtained in coupled atmosphere ocean models.

Acknowledgments. We are grateful to Annalisa Cherchi and Silvio Gualdi for discussions on the ENSO phenomenon, its teleconnections, and modeling; and to Antonio Navarra for the discussion and careful reading of the manuscript and for suggesting improvements. We also would like to thank Sarah Ineson, Paul Kushner, Judith Perlwitz, and Adam Scaife for discussions during the preparation of this work, and three anonymous 
reviewers for their constructive comments, which have helped to improve this manuscript. We acknowledge the ECMWF, Special Project on Middle Atmosphere Modelling, for providing computing time for the new set of simulations presented here. Part of this work was supported by the SCOUT-O3 Integrated Project (Contract 505390) of the European Commission. Chiara Cagnazzo was supported by the Centro Euro-Mediterraneo per i Cambiamenti Climatici.

\section{REFERENCES}

Baldwin, M. P., and T. J. Dunkerton, 2001: Stratospheric harbingers of anomalous weather regimes. Science, 294, 581-584.

Boville, B. A., 1984: The influence of the polar night jet on the tropospheric circulation in a GCM. J. Atmos. Sci., 41, 11321142.

Brönnimann, S., 2007: Impact of El Niño-Southern Oscillation on European climate. Rev. Geophys., 45, RG3003, doi:10.1029/ 2006RG000199.

Camp, C. D., and K.-K. Tung, 2007: Stratospheric polar warming by ENSO in winter: A statistical study. Geophys. Res. Lett., 34, L04809, doi:10.1029/2006GL028521.

Charlton, A. J., A. O'Neill, W. A. Lahoz, and A. C. Massacand, 2004: Sensitivity of tropospheric forecasts to stratospheric initial conditions. Quart. J. Roy. Meteor. Soc., 130, 1771-1792.

—_, and Coauthors, 2007: A new look at stratospheric sudden warmings. Part II. Evaluation of numerical model simulations. J. Climate, 20, 470-488.

Fraedrich, K., and K. Müller, 1992: Climate anomalies in Europe associated with ENSO extremes. Int. J. Climatol., 12, 25-31.

Hoerling, M. P., A. Kumar, and M. Zhong, 1997: El Niño, La Niña, and the nonlinearity of their teleconnections. J. Climate, 10, 1769-1786.

Honda, M., H. Nakamura, J. Ukita, I. Kousaka, and K. Takeuchi, 2001: Interannual seesaw between the Auletian and Icelandic lows. Part I: Seasonal dependence and life cycle. J. Climate, 14, 1029-1042.

Kushner, P. J., and Coauthors, 2007: The SPARC DynVar Project: A SPARC project on the dynamics and variability of the coupled stratosphere-troposphere system. SPARC Newsletter, No. 29, SPARC Project Office, Toronto, Ontario, Canada, 9-14.

Labitzke, K., 1981: Stratospheric-mesospheric midwinter disturbances: a summary of observed characteristics. J. Geophys. Res., 86, 9665-9678.

, and H. van Loon, 1989: The Southern Oscillation. Part IX: The influence of volcanic eruptions on the Southern Oscillation in the stratosphere. J. Climate, 2, 1223-1226.
Manzini, E., M. A. Giorgetta, M. Esch, L. Kornblueh, and E. Roeckner, 2006: The influence of sea surface temperatures on the northern winter stratosphere: Ensemble simulations with the MAECHAM5 model. J. Climate, 19, 3863-3881.

Mathieu, P.-P., R. T. Sutton, B. Dong, and M. Collins, 2004: Predictability of winter climate over the North Atlantic European region during ENSO events. J. Climate, 17, 1953-1974.

Norton, W. A., 2003: Sensitivity of northern hemisphere surface climate to simulation of the stratospheric polar vortex. Geophys. Res. Lett., 30, 1627, doi:10.1029/2003GL016958.

Paul, J., F. Fortuin, and H. Kelder, 1998: An ozone climatology based on ozonesonde and satellite measurements. J. Geophys. Res., 103, 31 709-31 734.

Randall, D. A., and Coauthors, 2007: Climate models and their evaluation. Climate Change 2007: The Physical Science Basis, S. Solomon et al., Eds., Cambridge University Press, 633-640.

Reichler, T., P. J. Kushner, and L. M. Polvani, 2005: The coupled stratosphere-troposphere response to impulsive forcing from the troposphere. J. Atmos. Sci., 62, 3337-3352.

Roeckner, E., and Coauthors, 2006: Sensitivity of simulated climate to horizontal and vertical resolution in the ECHAM5 atmosphere model. J. Climate, 19, 3771-3791.

Scaife, A. A., J. R. Knight, G. K. Vallis, and C. K. Folland, 2005 A stratospheric influence on the winter NAO and North Atlantic surface climate. Geophys. Res. Lett., 32, L18715, doi:10.1029/2005GL023226.

Shaw, T. A., and T. G. Shepherd, 2007: Angular momentum conservation and gravity wave drag parameterization: Implications for climate models. J. Atmos. Sci., 64, 190-203.

Song, Y., and W. A. Robinson, 2004: Dynamical mechanisms for stratospheric influences on the troposphere. J. Atmos. Sci., 61, 1711-1725.

Taguchi, M., and D. L. Hartmann, 2006: Increased occurrence of stratospheric sudden warmings during El Niño as simulated by WACCM. J. Climate, 19, 324-332.

Thompson, D. W. J., and J. M. Wallace, 1998: The Arctic Oscillation signature in the wintertime geopotential height and temperature fields. Geophys. Res. Lett., 25, 1297-1300.

— and - 2000: Annular modes in the extratropical circulation. Part I: Month-to-month variability. J. Climate, 13, 10001016.

— J J. C. Futardo, and T. G. Shepherd, 2006: On the tropospheric response to anomalous stratospheric wave drag and radiative heating. J. Atmos. Sci., 63, 2616-2629.

van Oldenborgh, G. J., 2005: Comments on "predictability of winter climate over the North Atlantic European region during ENSO events." J. Climate, 18, 2770-2772.

Xie, P., and P. A. Arkin, 1997: Global precipitation: A 17-year monthly analysis based on gauge observations, satellite estimates, and numerical model outputs. Bull. Amer. Meteor. Soc., 78, 2539-2558. 